\title{
The rescue strategy of the search for a losing aircraft by planes
}

\author{
An Wang ${ }^{1, a}$ \\ ${ }^{1}$ North China Electric Power University, BaoDing 071000, China; \\ a15733228661@163.com
}

Keywords: Probability, The Number and the Distribution of wreckages

\begin{abstract}
This paper solves the problem based on the accident of the lost Malaysian flight MH370. Because lacking of the flight data after the plane losing contact, we define an enough big object searching region. According to the search area all the recue planes can search one day and the distribution of the wreckages, we divides the region into several parts, build an math model to find an order strategy to rescue each areas.
\end{abstract}

\section{Introduction}

With the rapid development of the transport, more and more people would like to travel around the world. However, the news about the airplane crashes becomes more and more frequent nowadays. which makes our heart up and down. Typically, the lost Malaysian flight MH370 is still not been found yet. Although there are several methods have been found, it's a big challenge to search for a lost plane without any flight data.

\section{Region division}

We lack the flight data after the plane losing contact, what's more, the black box does not have power to send signals. It's difficult to know the accurate location of the crashed plane. So,we define an enough big object searching area, the possibility that the plane crashes in it is $100 \%$. And we use the symbol $\Omega$ to stand for the area. To find the plane, we need a more precise area. We call it the key area. According to the number of debris, wreckage location, ocean currents and wind direction, we can find some suspected crash sites.

After consult material, we find the rescue plane usually carry out the parallel scanning mode as Figure 1.
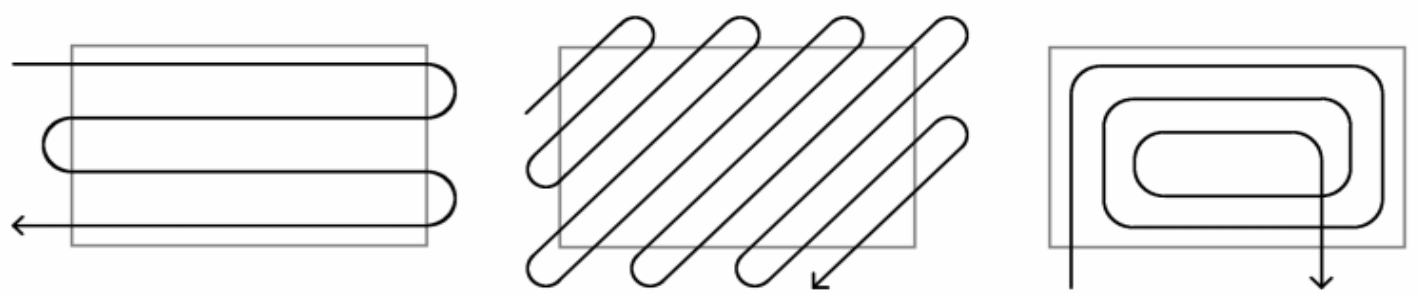

Figure 1: parallel scanning mode

In this way, we can maximize the search area with the minimum time. We think that the searching planes can explore a size of $s_{1}$ a day and the target region $\Omega$ sized $S_{\Omega}$ can be found by the satellite. We divide the part to $(n+1)$ parts.

$$
n=\left[\frac{S_{\Omega}}{S_{1}}\right]
$$

Then we can see the target area will be devided to 12 pieces. It's shown as Figure 2, Each of their size is equal to the area rescued planes searched a day. 


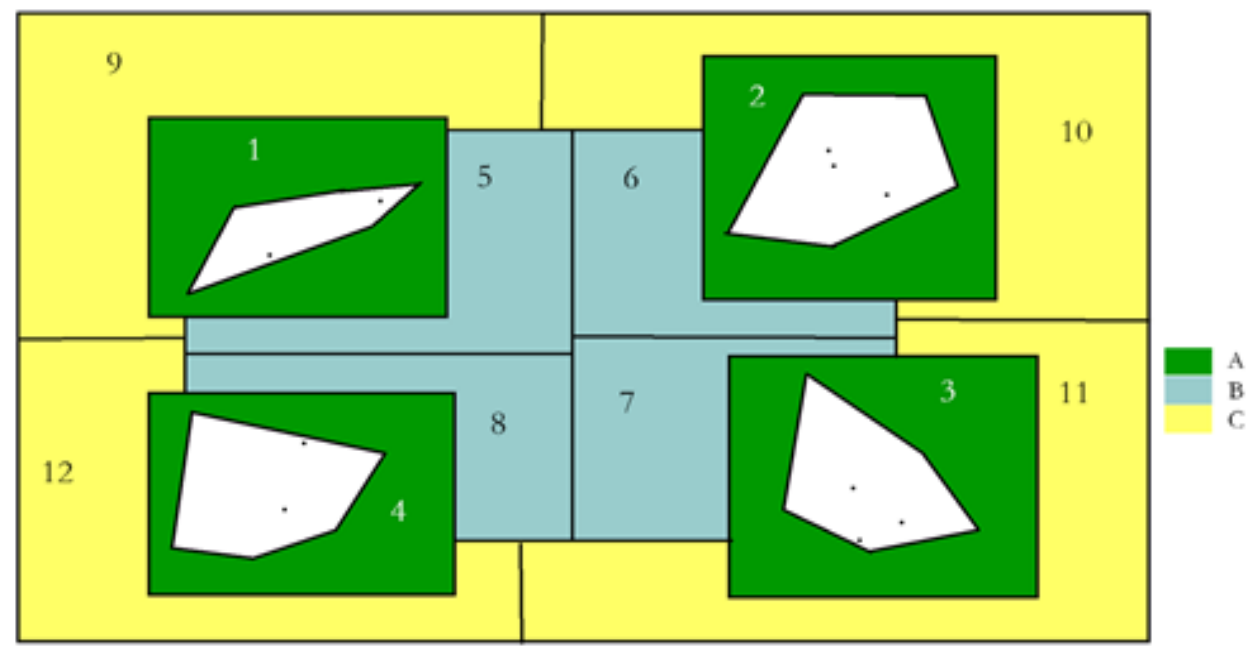

Figure 2: Region division for recusing

In the above picture, we define $1,2,3,4$ area as the part $A$,which has a white figure in the middle. The white figures in the middle are the envelope area of the crashed plane wreckage. When the number of the wreckages in the part is less than 3, we can't obtain the envelope graph. We define $5,6,7,8$ area as the part $B$, where is called the surrounding area of the plane wreckage envelope. And the area 9,10,11,12 is the edge part $C$ of the satellite searching. When we search the divided areas, we want the area include more wreckages. Therefore, Part $C$ has the minimum possibility and Part A has the maximum possibility to find the black box.

\section{Probability Analysis}

Through Analytic Hierarchy Process,we can get the probability the black box in the Part $A$ is about $40 \%$, the probability in the Part $B$ is about $35 \%$ and the probability in the Part $C$ is about $25 \%$. Part $C$ and Part $B$ follow evenly distribution. Therefore, the probability of each area numbered 5 to 8 is 0.0875 , the probability of areas numbered 9 to 12 is 0.0625 . But, the probability of each area in Part $A$ have positive correlation with the number and the convex hull's area of the wreckages inside.

Through the statistics and the calculation of the software, we can get a number array $N$ and an area array about the wreckages in each area, as:

$$
\begin{aligned}
& N=[4,9,17,13] \\
& S=[9413.99,11897.79,4905.04,4415.2]
\end{aligned}
$$

Then, we normalize the arraies, as:

$$
\begin{aligned}
& \omega_{S}=\frac{\left[s_{1}, s_{2} \ldots s_{n}\right]}{\sum_{i=1}^{n} s_{i}} \\
& \omega_{N}=\frac{\left[n_{1}, n_{2} \ldots n_{n}\right]}{\sum_{i=1}^{n} n_{i}}
\end{aligned}
$$

And we can get:

$$
\begin{aligned}
& \omega_{S}=[0.3073,0.3884,0.1601,0.1441] \\
& \omega_{N}=[0.093,0.2093,0.3953,0.3023]
\end{aligned}
$$

The probability weight array are the mean of

$$
\omega=\frac{\omega_{s}+\omega_{N}}{2}=[0.2002,0.2989,0.2777,0.2232]
$$


According to the probability weight, finally, we can an probability array as:

$p=[0.0801,0.1111,0.1196,0.0893,0.0875,0.0875,0.0875,0.0875,0.0625,0.0625,0.0625,0.0625]$

\section{The strategy of rescuing}

\section{The theoretical analysis}

Due to the ocean current moves differently at the bottom and the planes' recue abilities are limited, the area recued plane sesearched still have the posssibility to research again.

After consulting some material, the black box in an area but the plane undiscovered's probability $b$ can be caculated as $[1,2]$

$$
b=e^{-\frac{\omega}{s} z}
$$

Where $Z$ is the distance planes flied a day totally, $\omega$ is the average search width of planes, $s$ is the total area of the region to be recued.

After searching the area $\Theta$, the probability the black box exists in it can be caculated as:

$$
p_{\Theta}^{\prime}=p_{\Theta} \cdot e^{-\frac{\omega}{s} z}
$$

Where $p_{i}$ is the probability the black box exists in before rescue planes searched.

And other areas' probability can be caculated as

$$
\mu=\frac{1-p_{i}^{\prime}}{1-p_{i}} \quad p_{i}^{\prime}=p_{i} \cdot \mu \quad(i \neq \Theta)
$$

Reorder the areas according to their probabilities at the end of the day, the planes will choose the biggest one as the next day's target until all the area have been searched.

\section{Simulation}

After Flight ABCD crashes, the satellite provide a region whose area is about 246 thousand square kilometers for rescuing. We assume there are 12 rescue planes. They can travel $23100 \mathrm{NM}$ totally every day, their average search width is $10 \mathrm{NM}$. Through calculating through Matlab, we can get the order strategy as: $3-3-4-5-6-7-8-3-1-4-2-5-6-7-8-9-10-11-12$

\section{Conclusion}

We can see from our simulation, the area 3 are checked 3 times, the area 4 are checked 2 times. It totally cost 19 days to search all the region. The areas we first search are all the area contained wreckages. The areas we last searched are all the areas at the edges of the recue region. The strategy we get is reasonable. The model we built is utilizable. But our model doesn't deal with the situation that several transports searching together, which still need to be improved.

\section{References}

[1]Felix R,Ronald L.Space track report No.3:models for propagation of NORAD element sets[R].Peterson:Aerospace Defense Command,United States Air Force, 1980

[2]Levit C,Marshall W.Improved orbit predictions using two-line elements[J].Advances in Space Research,2011,47(7):1107-1115

[3]Wang Zhengming,Yi Dongyun.Instrumental data modeling and parameter estimation[M].Changsha:University of National Defence Technology Press,1996(in Chinese)

[4] Don R.Swanson Neil Smalheiser . Implicit text linkages between medline records: using arrowsmith as an aid to scientific discovery 1999,1(1). 